\title{
CRITERIA FOR DICHOTOMY OF LINEAR IMPULSIVE DIFFERENTIAL EQUATIONS
}

\author{
P.S. SIMEONOV AND D.D. BAINOV
}

\begin{abstract}
In the present paper necessary and sufficient conditions for $\left(\mu_{1}, \mu_{2}\right)$ dichotomy of linear impulsive differential equations are obtained without imposing conditions of bounded growth on these equations. The apparatus of piecewise continuous Lyapunov's functions is used.
\end{abstract}

\section{Introduction}

Let $\mathbb{Z}$ be the set of all integers. $S$ be the set of real or complex numbers, and let $J=\left(\omega_{-}, \omega_{+}\right) \subset \mathbb{R}$ be a real interval which can be bounded or unbounded. Consider the linear impulsive differential equations

$$
\begin{aligned}
x^{\prime}=A(t), & t \neq \tau_{k}, \\
x^{+}=A_{k} x, & t=\tau_{k},
\end{aligned}
$$

where $x \in S^{n}, t \in J, k \in \mathbb{Z}, A_{k} \in S^{n \times n}$ is an $n \times n$-matrix with entries of $S$ and the moments $\tau_{k}$ of impulse effect satisfy the conditions

$$
\lim _{k \rightarrow \pm \infty} \tau_{k}=\omega_{ \pm}, \quad \tau_{k}<\tau_{k+1} \quad(k \in \mathbb{Z})
$$

Denote by $P C\left(J, S^{n \times m}\right)$ the space of functions $f: J \rightarrow S^{n \times m}$ which are continuous for $t \neq \tau_{k}$ and for $t=\tau_{k}$ they have discontinuities of the first kind and are continuous from the left. We shall recall [1] that by a solution of (1) we mean any function $x: J \rightarrow S^{n}$

Received September 30, 1992.

1991 Mathematics Subject Classification. 34A37.

Key words and phrases. Dichotomy, impulsive differential equations.

This investigation was supported by the Bulgarian Ministry of Science and Higher Education under Grant MM-7. 
which is differentiable for $t \neq \tau_{k}$ and satisfies the equation $x^{\prime}=A(t) x$ and for $t=\tau_{k}$ satisfies the conditions

$$
x\left(\tau_{k}^{-}\right) \stackrel{\text { def }}{=} \lim _{t \rightarrow \tau_{k}-0} x(t)=x\left(\tau_{k}\right), \quad x\left(\tau_{k}^{+}\right) \stackrel{\text { def }}{=} \lim _{t \rightarrow \tau_{k}+0} x(t)=A_{k} x\left(\tau_{k}\right) .
$$

Assume the following conditions fulfilled:

A. $A(t) \in P C\left(J, S^{n \times n}\right)$.

A.2. $\operatorname{det} A_{k} \neq 0(k \in \mathbb{Z})$.

Under this assumption, all solutions $x(t)$ of $(1)$ are defined in $J$ and form an $n$ dimensional space of solutions which we denote by $X$. Let $|\cdot|$ denote some norm in $S^{n}$ and also the corresponding matrix norm. Let $X(t)$ be a fundamental matrix of solutions of equation (1) and let the functions $\mu_{1}, \mu_{2} \in P C(J, \mathbb{R})$.

Definition 1. Equation (1) is said to have a $\left(\mu_{1}, \mu_{2}\right)$-dichotomy if there exist supplementary projections $P_{1}, P_{2}$ on $S^{n}$ such that

$$
\left|X(t) P_{i} X^{-1}(s)\right| \leq K_{i} \exp \left(\int_{s}^{t} \mu_{i}(\tau) d \tau\right) \quad(-1)^{i}(s-t) \geq 0, i=1,2,
$$

where $K_{1}, K_{2} \geq 1$ are constants.

In the case when $\mu_{1}, \mu_{2}$ are constants equation (1) is said to have an exponential dichotomy if $\mu_{1}<0<\mu_{2}$ and ordinary dichotomy if $\mu_{1}=\mu_{2}=0$.

Condition (2) is equivalent to the conditions

$$
\begin{gathered}
\left|X(t) P_{i} \xi\right| \leq L_{i} \exp \left(\int_{s}^{t} \mu_{i}(\tau) d \tau\right)\left|X(s) P_{i} \xi\right| \quad \text { if }(-1)^{i}(s-t) \geq 0, i=1,2, \\
\left|X(t) P_{i} X^{-1}(t)\right| \leq M_{i}
\end{gathered}
$$

for any vector $\xi \in S^{n}$, where $L_{i}, M_{i} \geq 1$ are constants.

If the projector $P_{i}$ has rank $k_{i}, i=1,2, k_{1}+k_{2}=n$, then condition (3) means that the space of solutions $X$ has two supplementary subspaces $X_{1}, X_{2}$ of dimensions $k_{1}, k_{2}$ such that

$$
\begin{array}{ll}
|x(t)| \leq L_{1} \exp \left(\int_{s}^{t} \mu_{1}(\tau) d \tau\right)|x(s)| \quad\left(t \geq s, \quad x \in X_{1}\right) \\
|x(t)| \leq L_{2} \exp \left(\int_{s}^{t} \mu_{2}(\tau) d \tau\right)|x(s)| \quad\left(s \geq t, \quad x \in X_{2}\right)
\end{array}
$$

Condition (4) means that the supplementary projectors $X(t) P_{i} X^{-1}(t)$ from $S^{n}$ onto the subspaces $S_{i}(t)=\left\{x(t) \in S^{n}: x \in X_{i}\right\}, i=1,2$ are bounded uniformly on $t \in J$, or equivalently, that the "angle" between the subspaces $S_{i}(t), i=1,2$ is bounded away from zero for $t \in J$ (of. [2], p.156).

Some criteria for exponential dichotomy are well known [3]. However, the sufficient conditions usually require equation (1) to have a bounded growth (of. [3], Lectures 1,6,8). 
In the present paper three necessary and sufficient conditions for $\left(\mu_{1}, \mu_{2}\right)$-dichotomy without such constraints on the growth are given.

The proofs of the theorems are close to those by J.S. Muldowney of [4]. As an apparatus piecewise continuous comparison functions are used, which were introduced in [5] for investigation of the stability of the solutions of the impulsive differential equations by Lyapunov's direct method.

\section{Preliminary notes.}

We shall give some definitions and notation to be used henceforth.

Definition 2[5]. The function $U: J \times S^{n} \rightarrow \mathbb{R} .(t, x) \rightarrow U(t, x)$ is said to belong to the class $V_{0}$ if:

1. $U$ is continuous and locally Lipschitz continuous with respect to $x$ in the domain $G_{k}=\left(\tau_{k}, \tau_{k+1}\right) \times S^{n}(k \in \mathbb{Z})$.

2. For any $k \in \mathbb{Z}$ and $x \in S^{n}$ there exist the finite limits

$$
U\left(\tau_{k}^{-}, x\right)=\lim _{\substack{(t, y) \rightarrow\left(\tau_{k}, x\right) \\(t, y) \in G_{k-1}}} U(t, y), \quad U\left(\tau_{k}^{+}, x\right)=\lim _{\substack{(t, y) \rightarrow\left(\tau_{k}, x\right) \\(t, y) \in G_{k}}} U(t, y)
$$

and $U\left(\tau_{k}^{-}, x\right)=U\left(\tau_{k}, x\right)$.

For the function $U \in V_{0}$ and $t \neq \tau_{k}, x \in S^{n}$ define

$$
\dot{U}(t, x)=\limsup _{h \rightarrow 0_{+}} \frac{1}{h}[U(t+h, x+h A(t) x)-U(t, x)]
$$

- upper right derivative of the function $U$ with respect to equation (1).

We shall recall [6] that if $x(t)$ is a solution of (1), $U \in V_{0}$ and $u(t)=U(t, x(t))$, then

$$
D^{+} u(t)=\dot{U}(t, x(t)) \quad\left(t \neq \tau_{k}\right)
$$

where $D^{+} u$ is the upper right Dini derivative of the function $u$.

Definition 3. The couple of functions $V_{i}(t, x) \in V_{0}, i=1,2$ is said to be $a d$ missible if for any $t \in J$ there exist supplementary projectors $Q_{1}(t), Q_{2}(t)$ of rank $k_{1}, k_{2}$ independent of $t$ such that

$$
\begin{gathered}
\left|Q_{i}(t)\right| \leq N_{i} \quad(i=1,2) \\
\left|Q_{i}(t) x\right|^{r} \leq V_{i}(t, x) \leq b_{i}\left|Q_{i}(t) x\right|^{r} \quad(i=1,2)
\end{gathered}
$$

for any $(t, x) \in J \times S^{n}$, where $N_{i}, b_{i}, r>0$ are constants.

When the admissible couple is given, i.e. the couple of projectors $Q_{i}(i=1,2)$ and the number $r$ are determined uniquely, we shall always choose for $N_{i}, b_{i}$ the least possible values for which (5) and (6) are satisfied. 
If $V_{1}(t, x)$ and $V_{2}(t, x)$ is an admissible couple and $\lambda=\left(\lambda_{1}, \lambda_{2}\right)$ where $\lambda_{i} \geq 0$, then we define

$$
V(\lambda ; t, x)=\lambda_{1} V_{1}(t, x)-\lambda_{2} V_{2}(t, x)
$$

\section{Main results}

Theorem 1. Let condition $(A)$ hold and let there exist an admissible couple $V_{1}(t, x), V_{2}(t, x)$ and real numbers $\ell_{1}, \ell_{2}$ such that $0 \leq \ell_{i} b_{i}<1, i=1,2$ and

$$
\begin{aligned}
\dot{V}(\lambda ; t, x) & \leq \rho_{\lambda}(t) V(\lambda ; t, x) & & \left(\text { if } V(\lambda ; t, x) \geq 0, t \neq \tau_{k}\right), \\
\dot{V}(\lambda ; t, x) & \leq \delta_{\lambda}(t) V(\lambda ; t, x) & & \left(\text { if } V(\lambda ; t, x) \leq 0, t \neq \tau_{k}\right), \\
V\left(\lambda ; \tau_{k}^{+}, A_{k} x\right) & \leq V\left(\lambda ; \tau_{k}, x\right) & & (k \in \mathbb{Z})
\end{aligned}
$$

for $\lambda=\left(1, \ell_{2}\right)$ and $\lambda=\left(\ell_{1}, 1\right)$, where $\rho_{\lambda}, \delta_{\lambda} \in P C(J, \mathbb{R})$ and $\rho_{\lambda}=r \mu_{1}$ if $\lambda=\left(1, \ell_{2}\right)$, $\delta_{\lambda}=r \mu_{2}$ if $\lambda=\left(\ell_{1}, 1\right)$.

Then equation (1) has a $\left(\mu_{1}, \mu_{2}\right)$-dichotomy.

Theorem 2. Let conditions $(A)$ hold and let a function $\rho \in P C(J, \mathbb{R})$ exist such that $\mu_{1} \leq \rho \leq \mu_{2}$, as well as an admissible couple $V_{1}(t, x), V_{2}(t, x)$ and real numbers $\ell_{1}, \ell_{2}, 0<\ell_{i} b_{i}<1, i=1,2$ such that

$$
\begin{gathered}
\dot{V}_{1}(t, x) \leq r \rho(t) V_{1}(t, x) \quad\left(\text { if } V_{1}(t, x) \geq \ell_{2} V_{2}(t, x), t \neq \tau_{k}\right), \\
\dot{V}_{2}(t, x) \geq r \mu_{2}(t) V_{2}(t, x) \quad\left(\text { if } V_{1}(t, x) \leq \ell_{2} V_{2}(t, x), t \neq \tau_{k}\right), \\
\dot{V}_{1}(t, x) \leq r \mu_{1}(t) V_{1}(t, x) \quad\left(\text { if } \ell_{1} V_{1}(t, x) \geq V_{2}(t, x), t \neq \tau_{k}\right), \\
\dot{V}_{2}(t, x) \geq r \rho(t) V_{2}(t, x) \quad\left(\text { if } \ell_{1} V_{1}(t, x) \leq V_{2}(t, x), t \neq \tau_{k}\right), \\
V_{1}\left(\tau_{k}^{+}, A_{k} x\right) \leq V_{1}\left(\tau_{k}, x\right) \quad(k \in \mathbb{Z}), \\
V_{2}\left(\tau_{k}^{+}, A_{k} x\right) \geq V_{2}\left(\tau_{k}, x\right) \quad(k \in \mathbb{Z}) .
\end{gathered}
$$

Then equation (1) has a $\left(\mu_{1}, \mu_{2}\right)$-dichotomy.

Theorem 3. Let conditions (A) hold and let equation (1) have a $\left(\mu_{1}, \mu_{2}\right)$ dichotomy. Then there exists an admissible couple $V_{1}(t, x), V_{2}(t, x)$ such that

$$
\begin{aligned}
\dot{V}_{1}(t, x) & \leq r \mu_{1}(t) V_{1}(t, x) & & \left(t \neq \tau_{k}\right), \\
\dot{V}_{2}(t, x) & \geq r \mu_{2}(t) V_{2}(t, x) & & \left(t \neq \tau_{k}\right), \\
V_{1}\left(\tau_{k}^{+}, A_{k} x\right) & \leq V_{1}\left(\tau_{k}, x\right) & & (k \in \mathbb{Z}), \\
V_{2}\left(\tau_{k}^{+}, A_{k} x\right) & \geq V_{2}\left(\tau_{k}, x\right) & & (k \in \mathbb{Z}),
\end{aligned}
$$

Corollary 1. Let conditions $(A)$ hold. Then:

(a) The condition given as sufficient for a $\left(\mu_{1}, \mu_{2}\right)$-dichotomy in Theorem 1 , are also necessary. 
(b) When $\mu_{1} \leq \mu_{2}$ the condition given as sufficient for a $\left(\mu_{1}, \mu_{2}\right)$-dichotomy in Theorem 2, are also necessary.

(c) The condition given as necessary for a $\left(\mu_{1}, \mu_{2}\right)$-dichotomy in Theorem 3 , are also sufficient.

Proof of Corollary 1 . Assertion (b) is obvious since if the admissible couple $V_{1}(t, x), V_{2}(t, x)$ satisfies condition (16)-(21), then it satisfies also the conditions of Theorem 2. Assertions (a) and (c) follow from the fact that the conditions of Theorem 3 imply the conditions of Theorem 1 with $\ell_{1}=\ell_{2}=0$. We shall just note that if $U_{2}(t, x)=-V_{2}(t, x)$, then condition (17) implies that $\dot{U}_{2}(t, x) \leq r \mu_{2}(t) U_{2}(t, x)$ for $(t, x) \in J \times S^{n}, t \neq \tau_{k}$. The proof of this assertion is carried out as in [4], that is why we omit it.

In the proof of Theorem 1 and Theorem 2 we shall use the following lemma.

Lemma 1 [4]. Suppose that $P_{i}, i=1,2$ and $Q_{i}, i=1,2$ are two couples of supplementary projectors in $S^{n}$ such that

$$
\left|Q_{i}\right| \leq N \quad(i=1,2)
$$

if $\tau<1$ is a number such that

$$
\tau\left|Q_{1} P_{1}\right| \geq\left|Q_{2} P_{1}\right|, \quad \tau\left|Q_{2} P_{2}\right| \geq\left|Q_{1} P_{2}\right|
$$

then

$$
\left|P_{i}\right| \leq 2 N \frac{1+\tau}{1-\tau} \quad(i=1,2)
$$

Proof of Theorem 1 . Let $t_{0} \in J$ and

$$
W(\lambda ; t, x)= \begin{cases}\exp \left(-\int_{t_{0}}^{t} \rho_{\lambda}(\tau) d \tau\right) V(\lambda ; t, x) & \text { if } V(\lambda ; t, x) \geq 0 \\ \exp \left(-\int_{t_{0}}^{t} \delta_{\lambda}(\tau) d \tau\right) V(\lambda ; t, x) & \text { if } V(\lambda ; t, x) \leq 0\end{cases}
$$

From (7)-(9) it follows that if $x \in X$ then

$$
\begin{gathered}
D^{+} W(\lambda ; t, x) \leq 0 \quad\left(t \neq \tau_{k}\right), \\
W\left(\lambda ; \tau_{k}^{+}, x\left(\tau_{k}^{+}\right)\right) \leq W\left(\lambda ; \tau_{k}, x\left(\tau_{k}\right)\right) \quad(k \in \mathbb{Z}) .
\end{gathered}
$$

Therefore, the function $W(\lambda ; t, x(t))$ is nonincreasing in $J$ if $x(t)$ is a solution of (1) and $\lambda=\left(1, \ell_{2}\right)$ or $\lambda=\left(\ell_{1}, 1\right)$. In particular, if $\tau \in J$ and $0 \neq x(\tau) \in Q_{1}(\tau) S^{n}$, then from (6) $V_{1}(\tau, x(\tau))>0, V_{2}(\tau, x(\tau))=0$ since $Q_{2}(\tau) x(\tau)=0$. Then

$$
W(\lambda ; t, x(t)) \geq W(\lambda ; \tau, x(\tau))=\lambda_{1} \exp \left(-\int_{t_{0}}^{\tau} \rho_{\lambda}(u) d u\right) V_{1}(\tau, x(\tau))>0 \quad(t \leq \tau) .
$$


Choose a sequence $\tau_{m} \in J, \tau_{m} \rightarrow \omega_{+}$. Then for each $m$ there exists a $k_{1}$-dimensional subspace of solutions of (1) for which $W(\lambda ; t, x(t))$ is nonnegative and nonincreasing in $\left(\omega_{-}, \tau_{m}\right]$. Let $Y_{m}(t)$ be an $n \times k_{1}$-matrix of solutions of $(1)$ whose columns span this subspace and let the columns of $Y_{m}\left(\tau_{0}\right)$ be orthonormal. From the compactness of the unit sphere in $S^{n}$ it follows that a subsequence of $Y_{m}\left(\tau_{0}\right)$ (without loss of generality the sequence itself) converges to a matrix $Y\left(\tau_{0}\right)$ whose $k_{1}$ columns are orthonormal. Thus $\lim _{m \rightarrow \infty} Y_{m}(t)=Y(t)$ for any $t \in J$, where $Y(t)$ is an $n \times k_{1}$-matrix of solutions of (1) which has rank $k_{1}$. If $\xi \in S^{k_{1}}, x_{m}(t)=Y_{m}(t) \xi$ and $x(t)=Y(t) \xi$, then $W\left(\lambda ; t, x_{m}(t)\right) \leq 0, \omega_{-}<t \leq \tau_{m}$ implies $W(\lambda ; t, x(t)) \leq 0, \omega_{-}<t<\omega_{+}$. These conclusion are also valid for $\lambda=\left(1, \ell_{2}\right)$ and for $\lambda=\left(\ell_{1}, 1\right)$. Thus, if $x$ belongs to the $k_{1}$-dimensional space

$$
X_{1}=\left\{x \in X: x(t)=Y(t) \xi, \quad \xi \in S^{k_{1}}\right\}
$$

of solution of (1), then

$$
\begin{array}{ll}
V_{1}(t, x(t))-\ell_{2} V_{2}(t, x(t)) \geq 0 & (t \in J), \\
\ell_{1} V_{1}(t, x(t))-V_{2}(t, x(t)) \geq 0 & (t \in J) .
\end{array}
$$

Therefore, if $x \in X_{1}$ and $\lambda=\left(1, \ell_{2}\right)$ or $\lambda=\left(\ell_{1}, 1\right)$, then

$$
W(\lambda ; t, x(t))=\exp \left(-\int_{t_{0}}^{t} \rho_{\lambda}(u) d u\right) V(\lambda ; t, x(t))
$$

and this function is nonincreasing in $J$. In particular, for $\lambda=\left(1, \ell_{2}\right)$

$$
V_{1}(t, x(t))-\ell_{2} V_{2}(t, x(t)) \leq \exp \left(\int_{s}^{t} r \mu_{1}(u) d u\right)\left[V_{1}(s, x(s))-\ell_{2} V_{2}(s, x(s))\right] \quad(t \geq s)
$$

which together with (21) implies

$$
\left(1-\ell_{1} \ell_{2}\right) V_{1}(t, x(t)) \leq \exp \left(\int_{s}^{t} r \mu_{1}(u) d u\right) V_{1}(s, x(s)) \quad(t \geq s)
$$

Since $b_{i} \geq 1$, then $0<\ell_{i}<1$. Thus $1-\ell_{1} \ell_{2}>0$ and from (6)

$$
\left|Q_{1}(t) x(t)\right| \leq b_{1}^{1 / r}\left(1-\ell_{1} \ell_{2}\right)^{-1 / r} \exp \left(\int_{s}^{t} \mu_{1}(u) d u\right)\left|Q_{1}(s) x(s)\right| \quad(t \geq s)
$$

From (6) and (21) it follows that

$$
\left(\ell_{1} b_{1}\right)^{1 / r}\left|Q_{1}(t) x(t)\right| \geq\left|Q_{2}(t) x(t)\right| \quad\left(t \in J, x \in X_{1}\right)
$$


thus

$$
\begin{aligned}
|x(t)| & =\left|Q_{1}(t) x(t)+Q_{2}(t) x(t)\right| \\
& \leq\left|Q_{1}(t) x(t)\right|+\left|Q_{2}(t) x(t)\right| \\
& \leq\left[1+\left(\ell_{1} b_{1}\right)^{1 / r}\right]\left|Q_{1}(t) x(t)\right| .
\end{aligned}
$$

This, together with $\left|Q_{1}(s) x(s)\right| \leq N_{1}|x(s)|$ (from (5) and (22)) yields

$$
|x(t)| \leq L_{1} \exp \left(\int_{s}^{t} \mu_{1}(u) d u\right)|x(s)| \quad\left(t \geq s, x \in X_{1}\right),
$$

where $L_{1}=b_{1}^{1 / r}\left(1-\ell_{1} \ell_{2}\right)^{-1 / r}\left[1+\left(\ell_{1} b_{1}\right)^{1 / r}\right] N_{1}$.

Similar arguments show that there exists a $k_{2}$-dimensional subspace $X_{2}$ of solutions of (1) such that

$$
\begin{aligned}
& \left(\ell_{2} b_{2}\right)^{1 / r}\left|Q_{2}(t) x(t)\right| \geq\left|Q_{1}(t) x(t)\right| \quad\left(t \in J, x \in X_{2}\right), \\
& |x(t)| \leq L_{2} \exp \left(\int_{s}^{t} \mu_{2}(u) d u\right)|x(s)| \quad\left(s \geq t, x \in X_{2}\right) .
\end{aligned}
$$

Since $0<\ell_{i} b_{i}<1$, then from inequalities (23) and (24) it follows that the spaces $X_{1}, X_{2}$ are supplementary. That is why from (24) and (26) it follows that there exist supplementary projectors $P_{1}, P_{2}$ in $S^{n}$ such that (4) is valid. Finally, (5), (23) and (25) show that the conditions of Lemma 1 are satisfied for any $t \in J$ for the projectors $Q_{i}(t)$, $P_{i}(t)=X(t) P_{i} X^{-1}(t)$ with $\tau=\max \left\{\left(\dot{\ell}_{1} b_{1}\right)^{1 / r},\left(\ell_{2} b_{2}\right)^{1 / r}\right\}$ and $N=\max \left\{N_{1}, N_{2}\right\}$. That is why (20) imlies that (4) holds.

Proof of Theorem 2. First we suppose that $\rho=0$. Let $x(t)$ be an arbitrary solution of (1). Then from (10) and (14) it follows that $V_{1}(t, x(t))$ is nonincreasing in the interval $I \subset J$ if $V_{1}(t, x(t)) \geq \ell_{2} V_{2}(t, x(t))$ for any $t \in \mathbb{I}$. Similary, from (13) and (15) it follows that $V_{2}(t, x(t))$ is nondecreasing in $I$ if $\ell_{1} V_{1}(t, x(t)) \leq V_{2}(t, x(t))$ for all $t \in I$.

First we shall show that if $\ell_{1} V_{1}(t, x(t))<V_{2}(t, x(t))$ for some $t=\tau \in J$, then there exists $\mu \in\left(\tau, \omega_{+}\right)$such that

$$
\ell_{1} V_{1}(t, x(t))<V_{2}(t, x(t)) \quad(t \in[\tau, \mu])
$$

In fact, if $\tau=\tau_{k}$, then (27) follows by continuity. If $\tau=\tau_{k}$, then from $\ell_{1} V_{1}\left(\tau_{k}, x\left(\tau_{k}\right)\right)<$ $V_{2}\left(\tau_{k}, x\left(\tau_{k}\right)\right)$ by $(14)$ and (15) it follows that

$$
\ell_{1} V_{1}\left(\tau_{k}^{+}, x\left(\tau_{k}^{+}\right)\right) \leq \ell_{1} V_{1}\left(\tau_{k}, x\left(\tau_{k}\right)\right)<V_{2}\left(\tau_{k}, x\left(\tau_{k}\right)\right) \leq V_{2}\left(\tau_{k}^{+}, x\left(\tau_{k}^{+}\right)\right)
$$

which, also by continuity, implies (27) for some $\mu>\tau$.

Now we claim that if $\ell_{1} V_{1}(\tau, x(\tau))<V_{2}(\tau, x(\tau))$ for $\tau \in J$, then $\ell_{1} V_{1}(t, x(t))<$ $V_{2}(t, x(t))$ for $t \in\left[\tau, \omega_{+}\right)$. Suppose that this is not true, i.e. that there exists $s>\mu$ such 
that $\ell_{1} V_{1}(s, x(s)) \geq V_{2}(s, x(s))$. Let $s_{0}$ be the infimum of the numbers $s$ enjoying this property. Then $s_{0} \geq \mu>\tau$ and

$$
\begin{gathered}
\ell_{1} V_{1}\left(s_{0}^{+}, x\left(s_{0}^{+}\right)\right) \geq V_{2}\left(s_{0}^{+}, x\left(s_{0}^{+}\right)\right), \\
\ell_{1} V_{1}(t, x(t))<V_{2}(t, x(t)) \quad\left(t \in\left[\tau, s_{0}\right)\right),
\end{gathered}
$$

whence by continuity from the left

$$
\ell_{1} V_{1}\left(s_{0}, x\left(s_{0}\right)\right) \leq V_{2}\left(s_{0}, x\left(s_{0}\right)\right) .
$$

We have that

$$
V_{1}\left(s_{0}, x\left(s_{0}\right)\right)<\ell_{2} V_{2}\left(s_{0}, x\left(s_{0}\right)\right) .
$$

Otherwise, $V_{1}\left(s_{0}, x\left(s_{0}\right)\right) \geq \ell_{2} V_{2}\left(s_{0}, x\left(s_{0}\right)\right)$ and by (30)

$$
V_{2}\left(s_{0}, x\left(s_{0}\right)\right) \geq \ell_{1} V_{1}\left(s_{0}, x\left(s_{0}\right)\right) \geq \ell_{1} \ell_{2} V_{2}\left(s_{0}, x\left(s_{0}\right)\right),
$$

whence it follows that $V_{2}\left(s_{0}, x\left(s_{0}\right)\right)=0$ and $x\left(s_{0}\right)=0$ (by (30) and (6)) which is impossible.

From (31) and the continuity from the left of $x(t)$ it follows that there exists $\eta<s_{0}$ such that

$$
V_{1}(t, x(t))>\ell_{2} V_{2}(t, x(t)) \quad\left(t \in\left[\eta, s_{0}\right]\right) .
$$

Then in the interval $J_{1}=\left[\eta, s_{0}\right] \cap\left[\tau, s_{0}\right]$ the function $V_{1}(t, x(t))$ is nonincreasing and the function $V_{2}(t, x(t))$ is nondecreasing and for $t \in J_{1}$ by (14), (28) and (15) we have

$$
\begin{aligned}
\ell_{1} V_{1}(t, x(t)) & \geq \ell_{1} V_{1}\left(s_{0}, x\left(s_{0}\right)\right) \geq \ell_{1} V_{1}\left(s_{0}^{+}, x\left(s_{0}^{+}\right)\right) \geq V_{2}\left(s_{0}^{+}, x\left(s_{0}^{+}\right)\right) \\
& \geq V_{2}\left(s_{0}, x\left(s_{0}\right)\right) \geq V_{2}(t, x(t)),
\end{aligned}
$$

which contradicts (29). Thus the assertion is proved. It implies that if

$$
\ell_{1} V_{1}(t, x(t)) \geq V_{2}(t, x(t))
$$

is valid for $t=\tau$, then it is also valid for $t \in\left(\omega_{-}, \tau\right]$.

If the assumption $\rho=0$ is not valid, then the assertion in relation to (32) can be proved in the same way if in the proof we replace

$V_{i}(t, x)$ by $\exp \left(\int_{t_{0}}^{t} r \rho(u) d u\right) V_{i}(t, x), i=1,2$.

As in the proof of Theorem 1, considering a sequence $\tau_{m} \rightarrow \omega_{+}$we prove that there exists a $k_{1}$-dimensional subspace $X_{1}$ of solutions of (1) such that (32) is valid for all $t \in J$ and $x \in X_{1}$. From (6) and (32) we conclude that (23) is valid for each $x \in X_{1}$ and from (6), (10)-(15), (32) - that (24) is valid for each $x \in X_{1}$ with $L_{1}=b_{1}^{1 / r}\left[1+\left(\ell_{1} b_{1}\right)^{1 / r}\right] N_{1}$. Analogous arguments show the existence of a $k_{2}$-dimensional subspace $X_{2}$ of solutions of (1) satisfying (25) and (26), which completes the proof. 
Proof of Theorem 3. Suppose that (1) has a $\left(\mu_{1}, \mu_{2}\right)$-dichotomy and let

$$
\begin{aligned}
& V_{1}(t, x)=\sup _{\tau \geq t}\left|X(\tau) P_{1} X^{-1}(t)\right| \exp \left(-\int_{t}^{\tau} \mu_{1}(u) d u\right), \\
& V_{2}(t, x)=\sup _{\tau<t}\left|X(\tau) P_{2} X^{-1}(t)\right| \exp \left(-\int_{t}^{\tau} \mu_{2}(u) d u\right),
\end{aligned}
$$

for each $(t, x) \in J \times S^{n}$, where $X(t)$ and $P_{i}$ are as in (3) and (4).

First we shall show that the relations (5), (6) hold with $r=1$ and $Q_{i}(t)=$ $X(t) P_{i} X^{-1}(t), i=1,2$. In fact, (4) implies immediately that $\left|Q_{i}(t)\right| \leq M_{i}, t \in J$. From the definitions of $V_{i}(t, x), i=1,2$ and the continuity from the left of $X(\tau)$ it follows that

$$
\left|Q_{i}(t) x\right|=\left|X(t) P_{i} X^{-1}(t) x\right| \leq V_{i}(t, x), \quad i=1,2
$$

and from (4) with $\xi=X^{-1}(t) x$ we have

$$
\begin{aligned}
\left|X(\tau) P_{i} X^{-1}(t) x\right| & \leq L_{i} \exp \left(\int_{t}^{\tau} \mu_{i}(u) d u\left|X(t) P_{i} X^{-1}(t) x\right|\right. \\
& =L_{i} \exp \left(\int_{t}^{\tau} \mu_{i}(u) d u\right)\left|Q_{i}(t) x\right| \quad\left((-1)^{i}(t-\tau) \geq 0\right) .
\end{aligned}
$$

That is why

$$
V_{i}(t, x) \leq L_{i}\left|Q_{i}(t) x\right|, \quad i=1,2
$$

with which (5), (6) are proved.

For $t \in J$ and $x, y \in S^{n}$ we have

$$
\begin{aligned}
& \left|V_{1}(t, x)-V_{1}(t, y)\right| \\
= & \left|\sup _{\tau \geq t}\right| X(\tau) P_{1} X^{-1}(t) x\left|e^{-\int_{t}^{\tau} \mu_{1}}-\sup _{\tau \geq t}\right| X(\tau) P_{1} X^{-1}(t) y\left|e^{-\int_{t}^{\tau} \mu_{1}}\right| \\
\leq & \sup _{\tau \geq t}\left|X(\tau) P_{1} X^{-1}(t)(x-y)\right| e^{-\int_{t}^{\tau} \mu_{1}} \\
= & V_{1}(t, x-y) \leq L_{1}\left|Q_{1}(t)(x-y)\right| \leq L_{1} M_{1}|x-y|,
\end{aligned}
$$

i.e. $V_{1}(t, x)$ is Lipschitz continuous in $x$. Analogously it is proved that $V_{2}(t, x)$ is also Lipschitz continuous in $x$.

Let $t \in\left(\tau_{k} \tau_{k+1}\right), x \in S^{n}$ and $0<\delta<\min \left(\tau_{k+1}-t, t-\tau_{k}\right)$. Then

$$
\begin{aligned}
\left|V_{1}(t+\delta, y)-V_{1}(t, x)\right| \leq & \left|V_{1}(t+\delta, y)-V_{1}(t+\delta, x)\right| \\
& +\mid V_{1}(t+\delta, x)-V_{1}\left(t+\delta, X\left(t+\delta, X(t+\delta) X^{-1}(t) x\right) \mid\right. \\
& \left|V_{1}\left(t+\delta, X(t+\delta) X^{-1}(t) x\right)-V_{1}(t, x)\right| .
\end{aligned}
$$

The first two addends in (33) are small when $\delta$ and $|x-y|$ are small since $V_{1}(t, x)$ is Lipschitz continuous in $x$. If for $\delta \geq 0$ we set

$$
a(\delta)=\sup _{\tau \geq t+\delta}\left|X(\tau) P_{1} X^{-1}(t) x\right| e^{-\int_{t}^{r} \mu_{1}}
$$


then a straightforward verification shows that

$$
\left|V_{1}\left(t+\delta, X(t+\delta) X^{-1}(t) x\right)-V_{1}(t, x)\right|=\left|a(\delta) e^{\int_{t}^{t+\delta} \mu_{1}}-a(0)\right|
$$

Since the function $a(\delta)$ is nonincreasing for $\delta \geq 0$ and $a(\delta) \rightarrow a(0)$ as $\delta \rightarrow 0+$, then (33) and (34) imply the continuity of $V_{1}(t, x)$ in the set $G_{k}, k \in \mathbb{Z}$. Analogously the continuity of $V_{2}(t, x)$ in $G_{k}, k \in \mathbb{Z}$ is proved.

Let $x(t)$ be a solution of $(1)$ and $h>0$. Then for $t \neq \tau_{k}$

$$
\begin{aligned}
V_{1}(t+h, x(t+h)) & =\sup _{\tau \geq t+h}\left|X(\tau) P_{1} X^{-1}(t+h) x(t+h)\right| e^{-\int_{i+h}^{\tau} \mu_{1}} \\
& =\sup _{\tau \geq t+h}\left|X(\tau) P_{1} X^{-1}(t) x(t)\right| e^{-\int_{t+h}^{\tau} \mu_{1}} \\
& \leq \sup _{\tau \geq t}\left|X(\tau) P_{1} X^{-1}(t) x(t)\right| e^{-\int_{t}^{\tau} \mu_{1}} \cdot e^{\int_{t}^{t+h} \mu_{1}} \\
& =V_{1}(t, x(t)) e^{\int_{t}^{t+h} \mu_{1}} .
\end{aligned}
$$

therefore,

$$
\left.\frac{1}{h}\left[V_{1}(t+h), x(t+h)\right)-V_{1}(t, x(t))\right] \leq \frac{1}{h}\left[e^{\int_{t}^{t+h} \mu_{1}}-1\right] V_{1}(t, x(t)),
$$

i.e. $D^{+} V_{1}(t, x(t)) \leq \mu_{1}(t) V_{1}(t, x(t))$ which implies $\dot{V}_{1}(t, x) \leq \mu_{1}(t) V_{1}(t, x)$ since $V_{1}(t, x)$ is Lipschitz continuous in $x$. Analogously we find

$$
D_{-} V_{2}(t, x(t)) \geq \mu_{2}(t) V_{2}(t, x(t))
$$

which implies $D^{+} V_{2}(t, x(t)) \geq \mu_{2}(t) V_{2}(t, x(t))$ since $V_{2}(t, x(t))$ and $\mu_{2}(t)$ are continuous for $t \neq \tau_{k}$. Thus

$$
\dot{V}_{2}(t, x) \geq \mu_{2}(t) V_{2}(t, x)
$$

with which (16) and (17) are proved.

Now we shall prove the existence of the limits $V_{i}\left(\tau_{k}^{+}, x\right)$ and $V_{i}\left(\tau_{k}^{-} x\right), i=1,2$. Let $t_{i} \in\left(\tau_{k}, \tau_{k+1}\right), x_{i} \in S^{n}, u_{i}=X\left(t_{i}\right) X^{-1}\left(\tau_{k}^{+}\right) x, i=1,2$. Then

$$
\begin{aligned}
\left|V_{1}\left(t_{1}, x_{1}\right)-V_{2}\left(t_{2}, x_{2}\right)\right| \leq & \left|V_{1}\left(t_{1}, x_{1}\right)-V_{1}\left(t_{1}, u_{1}\right)\right|+\left|V_{1}\left(t_{2}, x_{2}\right)-V_{1}\left(t_{2}, u_{2}\right)\right| \\
& +\left|V_{1}\left(t_{1}, u_{1}\right)-V_{1}\left(t_{2}, u_{2}\right)\right| .
\end{aligned}
$$

By the Lipschitz continuity of $V_{1}(t, x)$ in $x$

$$
\left|V_{1}\left(t_{i}, x_{i}\right)-V_{1}\left(t_{i}, u_{i}\right)\right| \leq L_{1}\left|x_{i}-u_{i}\right| \leq L_{1}\left(\left|x_{i}-x\right|+u_{i}-x \mid\right) .
$$


But $\left|u_{i}-x\right|=\left|X\left(t_{i}\right) X^{-1}\left(\tau_{k}^{+}\right) x-x\right| \rightarrow 0$ as $t_{i} \rightarrow \tau_{k}^{+}$. Therefore, the first two addends in (35) tend to zero as $\left(t_{i}, x_{i}\right) \rightarrow\left(\tau_{k}^{+}, x\right), i=1,2$. Moreover, if for $\delta>0$ we define

$$
a(\delta)=\sup _{\tau \geq \tau_{k}+\delta}\left|X(\tau) P_{1} X^{-1}\left(\tau_{k}\right) x\right| e^{-\int_{\tau_{k}}^{\tau} \mu_{1}}
$$

then

$$
\begin{aligned}
& \left|V_{1}\left(t_{1}, u_{1}\right)-V_{1}\left(t_{2}, u_{2}\right)\right| \\
= & \left|\sup _{\tau \geq t_{1}}\right| X(\tau) P_{1} X^{-1}\left(t_{1}\right) X\left(t_{1}\right) X^{-1}\left(\tau_{k}^{+}\right) x \mid e^{-\int_{t_{1}}^{\tau} \mu_{1}} \\
& -\sup _{\tau \geq t_{2}}\left|X(\tau) P_{1} X^{-1}\left(t_{2}\right) X\left(t_{2}\right) X^{-1}\left(\tau_{k}^{+}\right) x\right| e^{-\int_{t_{2}}^{\tau} \mu_{2}} \mid \\
= & \left|a\left(t_{1}-\tau_{k}\right) e^{\int_{\tau_{k}}^{t_{1}} \mu_{1}}-a\left(t_{2}-\tau_{k}\right) e^{\int_{\tau_{k}}^{t_{2}} \mu_{1}}\right|,
\end{aligned}
$$

i.e. the third addended in (35) tends to zero as $t_{i} \rightarrow \tau_{k}^{+}, i=1,2$. All this shows that the limit $V_{1}\left(\tau_{k}^{+}, x\right)$ exists. The existence of the other limits is proved analogously.

Now we can calculate

$$
\begin{aligned}
V_{1}\left(\tau_{k}^{+}, A_{k} x\right) & =\lim _{\nu \rightarrow \tau_{k}^{+}} V_{1}\left(\nu, X(\nu) X^{-1}\left(\tau_{k}^{+}\right) A_{k} x\right) \\
& =\lim _{\nu \rightarrow \tau_{k}^{+}} \sup _{\tau \geq \nu}\left|X(\tau) P_{1} X^{-1}(\nu) X(\nu) X^{-1}\left(\tau_{k}^{+}\right) A_{k} x\right| e^{-\int_{\nu}^{\tau} \mu_{1}} \\
& =\lim _{\nu \rightarrow \tau_{k}^{+}} \sup _{\tau \geq \nu}\left|X(\tau) P_{1} X^{-1}\left(\tau_{k}\right) x\right| e^{-\int_{\nu}^{\tau} \mu_{1}} \\
& =\sup _{r>\tau_{k}}\left|X(\tau) P_{1} X^{-1}\left(\tau_{k}\right) x\right| e^{-\int_{\tau_{k}}^{\tau} \mu_{1}} \leq V_{1}\left(\tau_{k}, x\right) \\
& =\sup _{\tau \geq \tau_{k}}\left|X(\tau) P_{1} X^{-1}\left(\tau_{k}\right) x\right| e^{-\int_{\tau_{k}}^{\tau} \mu_{1}}=V_{1}\left(\tau_{k}, x\right) \\
V_{2}\left(\tau_{k}^{-}, x\right) & =\lim _{\lambda \rightarrow \tau_{k}^{-}} V_{1}\left(\lambda, X(\lambda) X^{-1}\left(\tau_{k}\right) x\right) \\
& =\sup _{\tau \leq \tau_{k}}\left|X(\tau) P_{2} X^{-1}\left(\tau_{k}\right) x\right| e^{-\int_{\tau_{k}}^{\tau} \mu_{2}} \geq V_{2}\left(\tau_{k}, x\right) \\
V_{2}\left(\tau_{k}^{-}, x\right) & =\lim _{\lambda \rightarrow \tau_{k}^{-}} V_{2}\left(\lambda, X(\lambda) X^{-1}\left(\tau_{k}\right) x\right) \\
& =\sup _{\tau<\tau_{k}}\left|X(\tau) P_{2} X^{-1}\left(\tau_{k}\right) x\right| e^{-\int_{\tau_{k}}^{\tau} \mu_{2}}=V_{2}\left(\tau_{k}, x\right)
\end{aligned}
$$

Hence $V_{i}(t, x) \in V_{0}, i=1,2$ and (18), (19) are valid. Thus we completed the proof of Theorem 3 . 
Theorem 4. Let the matrix-valued functions $H_{i}(t) \in P C\left(J, S^{n}\right), i=1,2$ be Hermitian for each $t \in J$ and have derivatives $H_{i}^{\prime}(t) \in P C\left(J, S^{n}\right), i=1,2$. Let there exist constants $\ell_{i} \geq 0, b_{i} \geq 0, i=1,2$ such that $0 \leq \ell_{i} b_{i}<1$ and for any $t \in J$ :

(i) $H_{1}(t) H_{2}(t)=0$,

(ii) $H_{1}(t)+H_{2}(t) \geq I$,

(iii) $H_{i}(t) \leq b_{i} I, i=1,2$,

(iv) $H(\lambda ; t)=\lambda_{1} H_{1}(t)-\lambda_{2} H_{2}(t)$ satisfies

$H^{\prime}+A^{*} H+H A \leq 2 \mu_{1} H$ if $\lambda=\left(1, \ell_{2}\right), H_{1}-\ell_{2} H_{2} \geq 0, t \neq \tau_{k}$,

$H^{\prime}+A^{*} H+H A \leq 2 \mu_{2} H$ if $\lambda=\left(\ell_{1}, 1\right), \ell_{1} H_{1}-H_{2} \leq 0, t \neq \tau_{k}$,

(v) $A_{k}^{*} H_{i}\left(\tau_{k}^{+}\right) A_{k}=H_{i}\left(\tau_{k}\right), \quad i=1,2, k \in \mathbb{Z}$.

Then equation (1) has a $\left(\mu_{1}, \mu_{2}\right)$-dichotomy.

Proof. This theorem follows from Theorem 1. If rank $H_{i}(t)=k_{i}(t)$ then (i) implies nullity $H_{1}(t) \geq k_{2}(t)$ so that $k_{1}(t)+k_{2}(t) \leq n$ and (ii) imply $k_{1}(t)+k_{2}(t) \geq n$. Hence, $k_{1}(t)+k_{2}(t)=n$, which implies that $k_{1}, k_{2}$ are constants on each interval $\left(\tau_{k}, \tau_{k+1}\right]$ since these functions are lower semicontinuous on $\left(\tau_{k}, \tau_{k+1}\right], k \in \mathbb{Z}$. But from (v) we conclude that $\operatorname{rank} H_{i}\left(\tau_{k}^{+}\right)=\operatorname{rank} H_{i}\left(\tau_{k}\right)$ and therefore $k_{1}, k_{2}$ are constants in $J$. By (i) the matrix $H_{i}(t)$ commutes with $H_{1}(t)+H_{2}(t)$ thus $Q_{i}(t)=H_{i}(t)\left[H_{1}(t)+H_{2}(t)\right]^{-1}, i=1,2$ are supplementary Hermitian projectors of rank $k_{i}, i=1,2$ for each $t \in J$. The functions $V_{i}(t, x)=x^{*} H_{i}(t) x, i=1,2$ satisfy conditions (5), (6) and the conditions of Theorem 1. We omit the proof of this assertion since it is carried out as in [4]. Proposition 2.6. We shall only note that from (v) immediately follows that $V_{i}(t, x), i=1,2$ satisfy condition (g) of Theorem 1.

\section{References}

[1] D. D. Bainov and P. S. Simeonov, Stability Theory of Differential Equations with Impulse Effect: Theory and Applications, Ellis Horwood, 1989.

[2] Ju. L. Daleckii and M. G. Krein., "Stability of solutions of differential equations in Banach space," Amer. Math. Soc. Transl., 43 (1974).

[3] W. A. Coppel, "Dichotomices in Stability Theory," Lecture Notes in Math., vol. 629, SpringerVerlag, New York, 1978.

[4] J. S. Muldowney, "Dichotomies and asymptotic behaviour for linear differential systems," Transactions of the Amer. Math. Soc., vol 283, No 2 (1984), 465-484.

[5] P. S. Simeonov and D. D. Bainov., "Stability with respect to part of the variables in systems with impulse effect," J. Math. Anal. Appl. 117, No. 1 (1986), 247-263.

[6] T. Yoshizawa, Stability theory by Liapunov's second method, Math. Soc. Japan, Tokyo, 1966.

Plovdiv University, Paissii Hilendarski.

South-West University, Blagoevgrad, Bulgaria. 